\title{
Negative Imaginary Lemmas for Descriptor Systems
}

\author{
Junlin Xiong, Alexander Lanzon, and Ian R. Petersen
}

\begin{abstract}
This technical note studies the negative imaginary properties of descriptor linear systems based on state-space realizations. Under the assumption of a minimal realization, necessary and sufficient conditions are established to characterize the negative imaginary properties of descriptor systems in terms of linear matrix inequalities with equality constraints. In particular, a negative imaginary lemma, a strict negative imaginary lemma and a lossless negative imaginary lemma are developed. A multiple-input and multiple-output RLC circuit network is used as an illustrative example to validate the developed theory.
\end{abstract}

Index Terms-Descriptor systems, linear matrix inequalities, negative imaginary systems, RLC circuits.

\section{INTRODUCTION}

The negative imaginary property is often satisfied for linear dynamical systems stemming from flexible structures and passive linear electrical circuits [1], [2]. The question of how to characterise negative imaginary properties in terms of system matrices is one of the main research problems in the study of negative imaginary systems theory. Under the assumption of minimal state-space realizations, necessary and sufficient conditions have been established, and are summarized as the Negative Imaginary Lemma in [1], [3]. As a result, the negative imaginary properties can be numerically tested efficiently. Along this line of research, lossless and finite frequency negative imaginary lemmas have been proposed in [4], [5], respectively. The negative imaginary lemma in [1], [3] has also been generalized by removing the minimality assumption [6]. Furthermore, the definition of negative imaginary systems has been modified to symmetric transfer function matrices in [7], and to allow poles at the origin in [8]. Negative imaginary lemmas have been applied to the stability analysis of interconnected negative imaginary systems [1], [3], [9], [10], and to the synthesis of negative imaginary systems [2], [6].

In this technical note, we are interested in developing negative imaginary lemmas for descriptor systems. In practice, descriptor systems provide a convenient way to model many realistic systems, such as electrical circuits [11], and electric power grids [12]. In particular, descriptor state-space models can be readily obtained via modified nodal analysis [13], [14], compared to the standard state-space models, for large-scale RLC circuit networks. The research in this technical note is partially motivated by the model reduction problem of VLSI circuits, where some particular structure properties need to be preserved in the reduced models. For example, the passivity property is

Manuscript received October 15, 2013; revised July 16, 2014, January 28, 2015, and May 5, 2015; accepted May 20, 2015. Date of publication June 11, 2015; date of current version January 26, 2016. This work was supported by the NSFC (61374026), the EPSRC (EP/F06022X/1), the Royal Society, and the ARC. Recommended by Associate Editor T. Hagiwara.

J. Xiong is with the Department of Automation, University of Science and Technology of China, Hefei 230026, China (e-mail: junlin.xiong@ gmail.com).

A. Lanzon is with the Control Systems Centre, School of Electrical and Electronic Engineering, University of Manchester, Manchester M13 9PL, U.K. (e-mail: a.lanzon@ieee.org).

I. R. Petersen is with the School of Engineering and Information Technology, University of New South Wales at the Australian Defence Force Academy, Canberra ACT 2600, Australia (e-mail: i.r.petersen@ gmail.com).

Digital Object Identifier 10.1109/TAC.2015.2444233 one such property deserving preservation [11]. Because RLC circuits may exhibit the negative imaginary property by choosing appropriate inputs and outputs [2], it may also be desirable to preserve the negative imaginary property when the order of descriptor models is reduced. This expectation motivates the current study: how can we test negative imaginary properties directly from the descriptor state-space models? A simplified version of the negative imaginary lemma for descriptor systems has been proposed in [15], and the lemma there is based on the Weierstrass form of the system. Unfortunately, the Weierstrass form is usually difficult to obtain. Another criteria to test the negative imaginary property of descriptor systems is provided in [16], where the criteria is given in terms of a Kronecker canonical decomposition of a matrix pencil determined from the system matrices. Here, we aim to develop negative imaginary lemmas for descriptor systems without knowledge of the Weierstrass form, and in terms of the system matrices directly.

The main results of the technical note give negative imaginary lemmas for descriptor systems. A negative imaginary lemma for descriptor systems is derived under the assumption of minimal realizations. A strict negative imaginary lemma and a lossless negative imaginary lemma are also established in terms of system matrices based on a spectral factorization result. These lemmas provide necessary and sufficient conditions in terms of a set of LMIs with linear equality constrains, and these conditions are numerically efficient to test the negative imaginary properties of descriptor systems. When the descriptor systems reduce to standard linear systems, our results coincide with the results in the literature. The developed negative imaginary theory is validated by an example from multiple-input and multiple-output RLC networks, and can be applied to robust control of flexible structures and passive circuit networks.

Notation: Let $\mathbb{R}^{m \times n}$ and $\mathcal{R}^{m \times n}$ denote the set of $m \times n$ real matrices and real-rational proper transfer function matrices, respectively. $A^{T}$ and $A^{*}$ denote the transpose and the complex conjugate transpose of a complex matrix $A$, respectively. $R^{\sim}(s)$ represents the adjoint of transfer function matrix $R(s)$ and is given by $R^{T}(-s) . \Re[\cdot]$ is the real part of a complex number. The notation $X>0$ or $X \geq 0$, where $X$ is a real symmetric matrix, means that the matrix $X$ is positive definite or positive semidefinite.

\section{PRoblem Formulation}

Consider a class of dynamical systems described by

$$
\left\{\begin{array}{l}
E \dot{x}(t)=A x(t)+B u(t) \\
y(t)=C x(t)+D u(t)
\end{array}\right.
$$

where $x(t) \in \mathbb{R}^{n}$ is the system state, $u(t) \in \mathbb{R}^{m}$ is the control input and $y(t) \in \mathbb{R}^{m}$ is the measurement output. The matrices $E \in \mathbb{R}^{n \times n}$, $A \in \mathbb{R}^{n \times n}, B \in \mathbb{R}^{n \times m}, C \in \mathbb{R}^{m \times n}$ and $D \in \mathbb{R}^{m \times m}$ are constant matrices. The matrix $E$ is often a singular matrix. The pair $(E, A)$ is called regular if $\operatorname{det}(s E-A) \neq 0$ for some $s \in \mathbb{C}$; it is called stable if the roots of $\operatorname{det}(s E-A)=0$ lie in the left half of the complex plane. When $(E, A)$ is regular, the descriptor system (1) has a transfer function

$$
R(s)=C(s E-A)^{-1} B+D .
$$


The regularity of $(E, A)$ also ensures that there exist non-singular matrices $Q \in \mathbb{R}^{n \times n}, P \in \mathbb{R}^{n \times n}$ such that

$$
\begin{array}{rlr}
Q E P & =\left[\begin{array}{cc}
I & 0 \\
0 & N
\end{array}\right], & Q A P=\left[\begin{array}{cc}
A_{1} & 0 \\
0 & I
\end{array}\right] \\
Q B & =\left[\begin{array}{l}
B_{1} \\
B_{2}
\end{array}\right], & C P=\left[\begin{array}{ll}
C_{1} & C_{2}
\end{array}\right]
\end{array}
$$

where $N \in \mathbb{R}^{n_{2} \times n_{2}}$ is a nilpotent matrix, $A_{1} \in \mathbb{R}^{n_{1} \times n_{1}}, \quad B_{1} \in$ $\mathbb{R}^{n_{1} \times m}, B_{2} \in \mathbb{R}^{n_{2} \times m}, C_{1} \in \mathbb{R}^{m \times n_{1}}, C_{2} \in \mathbb{R}^{m \times n_{2}}$ and $n_{1}+n_{2}=$ $n$. The matrices on the right sides of (3) are called the Weierstrass form of the descriptor system (1).

Definition 1-[3]: A transfer function matrix $R(s) \in \mathcal{R}^{m \times m}$ is negative imaginary if

1) $R(s)$ has no poles at the origin and in $\Re[s]>0$;

2) $j\left[R(j \omega)-R^{*}(j \omega)\right] \geq 0$ for all $\omega \in(0, \infty)$ except values of $\omega$ where $j \omega$ is a pole of $R(s)$;

3) If $j \omega_{0}, \omega_{0} \in(0, \infty)$, is a pole of $R(s)$, it is at most a simple pole, and the residue matrix $K_{0} \triangleq \lim _{s \rightarrow j \omega_{0}}\left(s-j \omega_{0}\right) j R(s)$ is positive semidefinite Hermitian.

Remark 1: Both the negative imaginary and the positive real properties are concerned with the phase characteristics of transfer functions over all frequencies. In contrast, the bounded real property is a property about the gain characteristics of transfer functions over all frequencies [17]. For example, the transfer function $R_{1}(s)=b_{1} /(s+1)$ with $b_{1}>0$ is both negative imaginary and positive real for any $b_{1}>0$, and is bounded real with gain no greater than unity only for $b_{1} \leq 1$. The transfer function $R_{2}(s)=b_{2} /\left(s^{2}+1\right)$ with $b_{2}>0$ is always negative imaginary, and is neither positive real nor bounded real for any $b_{2}>0$. The transfer function $R_{3}(s)=b_{3} s /\left(s^{2}+1\right)$ with $b_{3}>0$ is only positive real.

Definition 2-[1]: A transfer function matrix $R(s) \in \mathcal{R}^{m \times m}$ is termed strictly negative imaginary if:

1) $R(s)$ has no poles in $\Re[s] \geq 0$;

2) $j\left[R(j \omega)-R^{*}(j \omega)\right]>0$ for $\omega \in(0, \infty)$.

Remark 2: Because negative imaginary properties (see Definition 1 and 2) are defined for transfer function matrices $R(s) \in \mathcal{R}^{m \times m}$, they are independent of the state-space realization of $R(s)$. As a result, the results expressed in terms of transfer functions in [1]-[4] are still valid for the transfer functions of descriptor systems.

The objective of the technical note is to develop state-space negative imaginary lemmas for descriptor systems.

\section{Necessary And Sufficient Negative IMAGINARY LEMMAS}

In this section, three negative imaginary lemmas are developed for descriptor systems under the minimal realization assumption of transfer functions. These lemmas extend the negative imaginary lemma (that is, [3, Lemma 7]), the strict negative imaginary lemma (that is, [3, Lemma 8]), the lossless negative imaginary lemma (that is, [4, Theorem 1]) to the descriptor systems case, respectively.

Assumption 1: The state-space realization $(E, A, B, C, D)$ is both controllable and observable.

In view of [18, Theorem 2-6.3], Assumption 1 is equivalent to that ( $E, A, B, C, D)$ is a minimal realization of the transfer function in (2); see also [19, Theorem 6.3]. The system (1) is controllable if and only if both $\left(A_{1}, B_{1}\right)$ and $\left(N, B_{2}\right)$ are controllable. It is observable if and only if both $\left(A_{1}, C_{1}\right)$ and $\left(N, C_{2}\right)$ are observable.

Now, we are ready to state our first result, which extends [3, Lemma 7] to the descriptor systems case.
Theorem 1: Consider a state-space realization $(E, A, B, C, D)$ of the transfer function $R(s) \in \mathcal{R}^{m \times m}$. Suppose Assumption 1 holds. Then $R(s)$ is negative imaginary if and only if:

1) $\operatorname{det}(A) \neq 0, R(\infty)=R^{T}(\infty)$;

2) there exist matrices $X \in \mathbb{R}^{n \times n}, Y \in \mathbb{R}^{n \times m}$ such that

$$
\begin{aligned}
A^{T} X+X^{T} A & \leq 0 \\
C^{T}+X^{T} E A^{-1} B & =A^{T} Y \\
E^{T} X=X^{T} E & \geq 0 \\
E^{T} Y & =0 .
\end{aligned}
$$

Proof: $(\Longrightarrow)$ Because the transfer function $R(s)$ has no poles at the origin, one has that the system matrix $A$ is non-singular. The nonsingularity of $A$ further implies that the pair $(E, A)$ is regular. Hence, the transfer function $R(s)$ has a Weierstrass form (3). Therefore, we have

$$
R(s)=C_{1}\left(s I-A_{1}\right)^{-1} B_{1}+D-C_{2} B_{2}-\sum_{i=1}^{h-1} s^{i} C_{2} N^{i} B_{2}
$$

where $h<n_{2}$ is the smallest integer such that $N^{h}=0$.

Because $R(s)$ is proper, which implies that $C_{2} N^{i} B_{2}=0$ for $i=$ $1,2, \ldots, h-1$, one has

$$
C_{2} N\left[\begin{array}{llll}
B_{2} & N B_{2} & \cdots & N^{n_{2}-1} B_{2}
\end{array}\right]=0 .
$$

Because $\left(N, B_{2}\right)$ is controllable, one has that $\left[\begin{array}{lll}B_{2} & N B_{2} & \cdots\end{array}\right.$ $\left.N^{n_{2}-1} B_{2}\right]$ is of full row rank. It follows from the above equality that $C_{2} N=0$. On the other hand, the observability of $\left(N, C_{2}\right)$ implies that

$$
\left[\begin{array}{c}
C_{2} \\
C_{2} N \\
\vdots \\
C_{2} N^{n_{2}-1}
\end{array}\right]
$$

is of full column rank. Therefore, $C_{2}$ must be of full column rank, which implies that $N=0$ from $C_{2} N=0$.

As a result, the transfer function $R(s)$ has a standard state-space realization

$$
R(s)=C_{1}\left(s I-A_{1}\right)^{-1} B_{1}+D-C_{2} B_{2} .
$$

Assumption 1 ensures that the state-space realization $\left(A_{1}, B_{1}, C_{1}\right.$, $\left.D-C_{2} B_{2}\right)$ is a minimal realization. In view of [3, Lemma 7], $R(s)$ is negative imaginary if and only if the following conditions hold:

1) $\operatorname{det}\left(A_{1}\right) \neq 0, D-C_{2} B_{2}=\left(D-C_{2} B_{2}\right)^{T}$;

2) there exists a matrix $X_{1} \in \mathbb{R}^{n_{1} \times n_{1}}, X_{1}=X_{1}^{T}>0$, such that

$$
\begin{aligned}
A_{1}^{T} X_{1}+X_{1} A_{1} & \leq 0 \\
C_{1}^{T}+X_{1} A_{1}^{-1} B_{1} & =0 .
\end{aligned}
$$

Considering the Weierstrass form (3), the first condition above is equivalent to the first condition in Theorem 1.

Now we prove that there exist matrices $X$ and $Y$ satisfying (4)-(7). Let

$$
X=Q^{T}\left[\begin{array}{cc}
X_{1} & 0 \\
0 & 0
\end{array}\right] P^{-1}, \quad Y=Q^{T}\left[\begin{array}{c}
0 \\
C_{2}^{T}
\end{array}\right]
$$

where the matrix $X_{1}=X_{1}^{T}>0$ satisfies (9) and (10). We will verify that $X$ and $Y$ satisfy (4)-(7). 
Equation (6) holds because of the following equivalences:

$$
\begin{aligned}
E^{T} X & =X^{T} E \geq 0 \\
& \Longleftrightarrow P^{T} E^{T} Q^{T} Q^{-T} X P=P^{T} X^{T} Q^{-1} Q E P \geq 0 \\
& \Longleftrightarrow\left[\begin{array}{cc}
X_{1} & 0 \\
0 & 0
\end{array}\right]=\left[\begin{array}{cc}
X_{1}^{T} & 0 \\
0 & 0
\end{array}\right] \geq 0 \\
& \Longleftrightarrow X_{1}=X_{1}^{T}>0 .
\end{aligned}
$$

Equation (7) follows from the equivalences:

$$
\begin{aligned}
E^{T} Y=0 & \Longleftrightarrow P^{T} E^{T} Q^{T} Q^{-T} Y=0 \\
& \Longleftrightarrow\left[\begin{array}{ll}
I & 0 \\
0 & 0
\end{array}\right]\left[\begin{array}{c}
0 \\
C_{2}^{T}
\end{array}\right]=0 .
\end{aligned}
$$

Equation (4) holds because

$$
\begin{array}{rl}
A^{T} & X+X^{T} A \leq 0 \\
& \Longleftrightarrow P^{T} A^{T} Q^{T} Q^{-T} X P+P^{T} X^{T} Q^{-1} Q A P \leq 0 \\
& \Longleftrightarrow\left[\begin{array}{cc}
A_{1}^{T} X_{1}+X_{1} A_{1} & 0 \\
0 & 0
\end{array}\right] \leq 0 \\
& \Longleftrightarrow A_{1}^{T} X_{1}+X_{1} A_{1} \leq 0 .
\end{array}
$$

Equation (5) is true due to

$$
\begin{aligned}
& C^{T}+X^{T} E A^{-1} B=A^{T} Y \\
& \Longleftrightarrow P^{T} C^{T}+P^{T} X^{T} Q^{-1} Q E P P^{-1} A^{-1} Q^{-1} Q B \\
&=P^{T} A^{T} Q^{T} Q^{-T} Y \\
& \Longleftrightarrow\left[\begin{array}{c}
C_{1}^{T}+X_{1} A_{1}^{-1} B_{1} \\
C_{2}^{T}
\end{array}\right]=\left[\begin{array}{c}
0 \\
C_{2}^{T}
\end{array}\right] \\
& \Longleftrightarrow C_{1}^{T}+X_{1} A_{1}^{-1} B_{1}=0 .
\end{aligned}
$$

$(\Longleftarrow)$ Because $\operatorname{det}(A) \neq 0$, the pair $(E, A)$ is regular. This implies that the Weierstrass form (3) exists for $R(s)$. In view of the properness of $R(s)$ and the controllability and observability assumption, one can conclude that $N=0$ by following the same lines as in the necessary part of the proof.

Suppose there exist matrices $X$ and $Y$ such that (4)-(7) hold. Let

$$
Q^{-T} X P=\left[\begin{array}{ll}
X_{1} & X_{2} \\
X_{3} & X_{4}
\end{array}\right], \quad Q^{-T} Y=\left[\begin{array}{c}
Y_{1} \\
Y_{2}
\end{array}\right]
$$

We will first verify that $X_{1}$ satisfies (9) and (10) and then prove $X_{1}=$ $X_{1}^{T}>0$.

Firstly, it follows from (6) that

$$
\begin{aligned}
E^{T} X & =X^{T} E \geq 0 \\
& \Longleftrightarrow\left[\begin{array}{cc}
X_{1} & X_{2} \\
0 & 0
\end{array}\right]=\left[\begin{array}{ll}
X_{1}^{T} & 0 \\
X_{2}^{T} & 0
\end{array}\right] \geq 0 \\
& \Longleftrightarrow X_{1}=X_{1}^{T} \geq 0, X_{2}=0 .
\end{aligned}
$$

Therefore

$$
Q^{-T} X P=\left[\begin{array}{cc}
X_{1} & 0 \\
X_{3} & X_{4}
\end{array}\right], \quad X_{1}=X_{1}^{T} \geq 0 .
$$

Secondly, it follows from (7) that

$$
E^{T} Y=0 \Longleftrightarrow P^{T} E^{T} Q^{T} Q^{-T} Y=0 \Longleftrightarrow Y_{1}=0 .
$$

Therefore

$$
Q^{-T} Y=\left[\begin{array}{c}
0 \\
Y_{2}
\end{array}\right]
$$

Inequality (9) follows from

$$
\begin{aligned}
A^{T} X+X^{T} A \leq 0 & \\
& \Longleftrightarrow\left[\begin{array}{cc}
A_{1}^{T} X_{1}+X_{1} A_{1} & X_{3}^{T} \\
X_{3} & X_{4}+X_{4}^{T}
\end{array}\right] \leq 0 \\
& \Longrightarrow A_{1}^{T} X_{1}+X_{1} A_{1} \leq 0 .
\end{aligned}
$$

Equality (10) holds because

$$
\begin{aligned}
C^{T} & +X^{T} E A^{-1} B=A^{T} Y \\
& \Longleftrightarrow\left[\begin{array}{c}
C_{1}^{T}+X_{1} A_{1}^{-1} B_{1} \\
C_{2}^{T}
\end{array}\right]=\left[\begin{array}{c}
0 \\
Y_{2}
\end{array}\right] \\
& \Longleftrightarrow C_{1}^{T}+X_{1} A_{1}^{-1} B_{1}=0, Y_{2}=C_{2}^{T} .
\end{aligned}
$$

Because we have shown that $X_{1}=X_{1}^{T} \geq 0$, we need to prove that $X_{1}$ is non-singular to obtain that $X_{1}=X_{1}^{T}>0$. Suppose that $X_{1}$ is singular. Then there exists an orthogonal matrix $U$ such that

$$
\begin{aligned}
U^{T} X_{1} U & =\left[\begin{array}{cc}
X_{11} & 0 \\
0 & 0
\end{array}\right], & U^{T} A_{1} U & =\left[\begin{array}{ll}
A_{11} & A_{12} \\
A_{13} & A_{14}
\end{array}\right] \\
U^{T} B_{1} & =\left[\begin{array}{c}
B_{11} \\
B_{12}
\end{array}\right], & C_{1} U & =\left[\begin{array}{ll}
C_{11} & C_{12}
\end{array}\right]
\end{aligned}
$$

where $X_{11}=X_{11}^{T}>0, U^{T} U=U U^{T}=I$. Also note that $A_{1}$ is nonsingular.

It follows from (9) that

$$
\begin{aligned}
& A_{1}^{T} X_{1}+X_{1} A_{1} \leq 0 \\
& \Longleftrightarrow U^{T} A_{1}^{T} U U^{T} X_{1} U+U^{T} X_{1} U U^{T} A_{1} U \leq 0 \\
& \Longleftrightarrow\left[\begin{array}{cc}
A_{11}^{T} X_{11}+X_{11} A_{11} & X_{11} A_{12} \\
A_{12}^{T} X_{11} & 0
\end{array}\right] \leq 0 \\
& \Longleftrightarrow A_{11}^{T} X_{11}+X_{11} A_{11} \leq 0, X_{11} A_{12}=0 \\
& \Longleftrightarrow A_{12}=0 \text { (because } X_{11} \text { is non-singular) } .
\end{aligned}
$$

Following from (10), we have

$$
\begin{aligned}
C_{1}^{T} & +X_{1} A_{1}^{-1} B_{1}=0 \\
& \Longleftrightarrow U^{T} C_{1}^{T}+U^{T} X_{1} U U^{T} A_{1}^{-1} U U^{T} B_{1}=0 \\
& \Longleftrightarrow\left[\begin{array}{c}
C_{11}^{T}+X_{11} A_{11}^{-1} B_{11} \\
C_{12}^{T}
\end{array}\right]=0 \\
& \Longrightarrow C_{12}=0 .
\end{aligned}
$$

Therefore, one has

$$
U^{T} A_{1} U=\left[\begin{array}{cc}
A_{11} & 0 \\
A_{13} & A_{14}
\end{array}\right], \quad C_{1} U=\left[\begin{array}{ll}
C_{11} & 0
\end{array}\right] .
$$

Hence

$$
\left[\begin{array}{c}
C_{1} \\
C_{1} A_{1} \\
\vdots \\
C_{1} A_{1}^{n_{1}-1}
\end{array}\right] U=\left[\begin{array}{c}
C_{1} U \\
C_{1} U U^{T} A_{1} U \\
\vdots \\
C_{1} U\left(U^{T} A_{1} U\right)^{n_{1}-1}
\end{array}\right]=\left[\begin{array}{cc}
C_{11} & 0 \\
C_{11} A_{11} & 0 \\
\vdots & \vdots \\
C_{11} A_{11}^{n_{1}-1} & 0
\end{array}\right] .
$$


This means that

$$
\operatorname{rank}\left(\left[\begin{array}{c}
C_{1} \\
C_{1} A_{1} \\
\vdots \\
C_{1} A_{1}^{n_{1}-1}
\end{array}\right]\right)<n_{1}
$$

which contradicts the observability assumption on $\left(A_{1}, C_{1}\right)$. Therefore, we have that $X_{1}$ is non-singular; hence $X_{1}>0$. Therefore, $R(s)$ is negative imaginary according to [3, Lemma 7]. This completes the proof.

Remark 3: Because the equality constraints in (5) and (7) are linear constraints, the solution set for (4)-(7) is a convex set. Free toolboxes such as YALMIP may be used to solve conditions (4)-(7) directly. Also, optimization techniques could be used to find a feasible solution to (4)-(7) by constructing the objective function from the equality constraints.

Remark 4: Theorem 1 can be considered as an extension of the negative imaginary lemma to the descriptor systems case. When $E=I,[3$, Lemma 7] can be recovered.

Remark 5: It follows from (8) that $R(\infty)=D-C_{2} B_{2}$, which seems dependent on the Weierstrass form (3). However, it follows from the sufficient part of the proof that if $X$ and $Y$ satisfy the equations in (4)-(7), then one has $Q^{-T} Y=\left[\begin{array}{c}0 \\ C_{2}^{T}\end{array}\right]$. This leads to $Y^{T} B=C_{2} B_{2}$. Therefore, we have $R(\infty)=D-Y^{T} B$. In other words, the condition $R(\infty)=R^{T}(\infty)$ in Theorem 1 can be replaced by $D-Y^{T} B=$ $\left(D-Y^{T} B\right)^{T}$.

The next result is an extension of [3, Corollary 1], and gives a spectral factorization of negative imaginary transfer function matrices. This result will be used to prove the strict negative imaginary lemma for descriptor systems.

Corollary 1: Consider a state-space realization $(E, A, B, C, D)$ of the transfer function $R(s) \in \mathcal{R}^{m \times m}$. Suppose Assumption 1 is satisfied. If $R(s)$ is negative imaginary, then there exists a real rational strictly proper transfer function matrix

$$
M(s)=L A^{-1} E(s E-A)^{-1} B
$$

such that

$$
R(s)-R^{\sim}(s)=-s M^{\sim}(s) M(s)
$$

for all $s$ with $s$ not a pole of $R(s)$. Here, $L \in \mathbb{R}^{n \times n}$ is a matrix satisfying $L^{T} L=-\left(A^{T} X+X^{T} A\right)$, where $X$ is a solution to conditions (4)-(7) in Theorem 1. In particular, one has

$$
j\left[R(j \omega)-R^{*}(j \omega)\right]=\omega M^{*}(j \omega) M(j \omega)
$$

for all $\omega \in[0, \infty)$ with $j \omega$ not a pole of $R(s)$.

Proof: Note that Assumption 1, together with the properness of $R(s)$, implies that $N=0$, as shown in the proof of Theorem 1. Let $L P=\left[\begin{array}{ll}L_{1} & L_{2}\end{array}\right]$. We first have that $M(s)$ is strictly proper by noting

$$
M(s)=L_{1} A_{1}^{-1}\left(s I-A_{1}\right)^{-1} B_{1} .
$$

Because $R(s)$ is negative imaginary, it follows from Theorem 1 that there exist two matrices $X, Y$ satisfying (5)-(7) and a matrix $L$ satisfying $A^{T} X+X^{T} A+L^{T} L=0$.

In a similar way to the sufficiency part of the proof of Theorem 1 , one can conclude that there exist matrices $X_{1} \in \mathbb{R}^{n_{1} \times n_{1}}, X_{1}=$ $X_{1}^{T}>0, L_{1} \in \mathbb{R}^{n_{1} \times n_{1}}$ such that

$$
A_{1}^{T} X_{1}+X_{1} A_{1}+L_{1}^{T} L_{1}=0 \text {, and } C_{1}^{T}+X_{1} A_{1}^{-1} B_{1}=0 .
$$

Let $\tilde{Y}_{1}=X_{1}^{-1}>0$ and $\tilde{L}_{1}=L_{1} X_{1}^{-1}$. The above conditions are equivalent to

$$
A_{1} \tilde{Y}_{1}+\tilde{Y}_{1} A_{1}^{T}+\tilde{L}_{1}^{T} \tilde{L}_{1}=0 \text {, and } B_{1}+A_{1} \tilde{Y}_{1} C_{1}^{T}=0 .
$$

Then we have

$$
M(s)=\tilde{L}_{1} \tilde{Y}_{1}^{-1} A_{1}^{-1}\left(s I-A_{1}\right)^{-1} B_{1} .
$$

Let $M_{1}(s)=\tilde{L}_{1} \tilde{Y}_{1}^{-1} A_{1}^{-1}\left(s I-A_{1}\right)^{-1} B_{1}$ and $R_{1}(s)=R(s)-$ $R(\infty)=C_{1}\left(s I-A_{1}\right)^{-1} B_{1}$. Then

$$
\begin{aligned}
R(s)-R^{\sim}(s) & =R(s)-R^{T}(-s) \\
& =\left[R_{1}(s)+R(\infty)\right]-\left[R_{1}(-s)+R(\infty)\right]^{T} \\
& =R_{1}(s)-R_{1}^{T}(-s)=R_{1}(s)-R_{1}^{\sim}(s) \\
& =-s M_{1}^{\sim}(s) M_{1}(s)=-s M^{\sim}(s) M(s)
\end{aligned}
$$

for all $s$ with $s$ not a pole of $R(s)$. The second last equality follows from [3, Corollary 1] and [3, Remark 6]. This proves that (12) holds. By letting $s=j \omega,(12)$ becomes

$$
R(j \omega)-R^{*}(j \omega)=-j \omega M^{*}(j \omega) M(j \omega) .
$$

Multiplying both sides of the above equation by $j$ leads to (13).

The next result is an extension of [3, Lemma 8], and gives a strict negative imaginary lemma for descriptor systems.

Theorem 2: Consider a state-space realization $(E, A, B, C, D)$ of the transfer function $R(s) \in \mathcal{R}^{m \times m}$. Suppose Assumption 1 is satisfied. Then $R(s)$ is strictly negative imaginary if and only if:

1) $(E, A)$ is stable, $R(\infty)=R^{T}(\infty)$;

2) there exist matrices $X \in \mathbb{R}^{n \times n}, Y \in \mathbb{R}^{n \times m}$ such that (4)-(7) hold;

3) $\operatorname{rank}(M(j \omega))=m$ for all $\omega \in(0, \infty)$, where $M(s)$ is defined in (11).

Proof: It can be seen from Definition 1 and Definition 2 that a strictly negative imaginary transfer function is also a negative imaginary one, but the converse is not true. Hence, the results in Theorem 1 and Corollary 1 are applicable to strictly negative imaginary transfer functions. The proof here will concentrate on the differences.

$(\Longrightarrow)$ It follows from Definition 2 that $R(s)$ has no poles in $\Re[s] \geq$ 0 . Therefore, the matrix $A_{1}$ in (3) is Hurwitz, and $(E, A)$ is stable.

Because $R(s)$ is strictly negative imaginary, it is also negative imaginary. According to Theorem 1 , we have that $R(\infty)=R^{T}(\infty)$ and the second condition holds.

Furthermore, in view of Corollary 1 and Definition 2, respectively, we have

$$
j\left[R(j \omega)-R^{*}(j \omega)\right]=\omega M^{*}(j \omega) M(j \omega)>0, \forall \omega \in(0, \infty) .
$$

Therefore, $M(j \omega)$ is of full column rank for $\omega \in(0, \infty)$.

$(\Longleftarrow)$ Firstly, the condition that $(E, A)$ is stable means that $R(s)$ has no poles in $\Re[s] \geq 0$. Secondly, in view of Theorem 1 , the first and second conditions in the theorem imply that $R(s)$ is negative imaginary. Then according to Corollary 1 , we have

$$
j\left[R(j \omega)-R^{*}(j \omega)\right]=\omega M^{*}(j \omega) M(j \omega), \quad \forall \omega \in(0, \infty) .
$$

Finally, the third condition implies that $M^{*}(j \omega) M(j \omega)>0$. Therefore $j\left[R(j \omega)-R^{*}(j \omega)\right]>0$ for all $\omega \in(0, \infty)$. Therefore, $R(s)$ is strictly negative imaginary.

The third condition in Theorem 2 can be replaced by other conditions as shown in the following lemma. 
Lemma 1: The third condition in Theorem 2 and the following statements are equivalent:

1) $\operatorname{rank}\left(R(j \omega)-R^{*}(j \omega)\right)=m$ for all $\omega \in(0, \infty)$.

2) $\operatorname{rank}\left(\left[\begin{array}{cc}A-j \omega E & B \\ L A^{-1} E & 0\end{array}\right]\right)=n+m$ for all $\omega \in(0, \infty)$.

3) $\operatorname{rank}\left(\left[\begin{array}{ccc}A-j \omega E & 0 & B \\ 0 & -A^{T}-j \omega E^{T} & C^{T} \\ C & B^{T} & D-D^{T}\end{array}\right]\right)=2 n+$ $m$ for all $\omega \in(0, \infty)$.

Proof: Condition 3 in Theorem $2 \Longleftrightarrow 1$ ). It follows from Corollary 1 that

$$
j\left[R(j \omega)-R^{*}(j \omega)\right]=\omega M^{*}(j \omega) M(j \omega), \quad \omega \in(0, \infty) .
$$

Hence $R(j \omega)-R^{*}(j \omega)$ is non-singular if and only if $M(j \omega)$ is of full column rank.

Condition 3 in Theorem $2 \Longleftrightarrow 2$ ). Note that

$$
\begin{aligned}
{\left[\begin{array}{cc}
A-j \omega E & B \\
L A^{-1} E & 0
\end{array}\right] } & =\left[\begin{array}{cc}
I & 0 \\
L A^{-1} E(A-j \omega E)^{-1} & I
\end{array}\right] \\
& \times\left[\begin{array}{cc}
A-j \omega E & 0 \\
0 & M(j \omega)
\end{array}\right]\left[\begin{array}{cc}
I & (A-j \omega E)^{-1} B \\
0 & I
\end{array}\right] .
\end{aligned}
$$

Hence

$$
\begin{aligned}
\operatorname{rank}\left(\left[\begin{array}{cc}
A-j \omega E & B \\
L A^{-1} E & 0
\end{array}\right]\right) & =\operatorname{rank}(A-j \omega E)+\operatorname{rank}(M(j \omega)) \\
& =n+\operatorname{rank}(M(j \omega)) .
\end{aligned}
$$

Therefore,

$$
\operatorname{rank}\left(\left[\begin{array}{cc}
A-j \omega E & B \\
L A^{-1} E & 0
\end{array}\right]\right)=n+m \Longleftrightarrow \operatorname{rank}(M(j \omega))=m .
$$

1) $\Longleftrightarrow 3)$. Firstly, note that

$$
\begin{aligned}
G(s) \triangleq & R(s)-R^{\sim}(s) \\
= & {\left[\begin{array}{ll}
C & B^{T}
\end{array}\right]\left(s\left[\begin{array}{cc}
E & 0 \\
0 & E^{T}
\end{array}\right]-\left[\begin{array}{cc}
A & 0 \\
0 & -A^{T}
\end{array}\right]\right)^{-1}\left[\begin{array}{c}
B \\
C^{T}
\end{array}\right] } \\
& +D-D^{T} .
\end{aligned}
$$

By noting $G(j \omega)=R(j \omega)-R^{*}(j \omega), \quad$ statement $\quad 1 \Longleftrightarrow$ $\operatorname{rank}(G(j \omega))=m \Longleftrightarrow$ statement 3. The last equivalence follows from applying the result of the equivalence between the third condition in Theorem 2 and the second statement in this lemma.

Next result extends [4, Theorem 1] to the descriptor systems case, and gives us a lossless negative imaginary lemma for descriptor systems.

Theorem 3: Consider a state-space realization $(E, A, B, C, D)$ of the transfer function $R(s) \in \mathcal{R}^{m \times m}$. Suppose Assumption 1 is satisfied. Then $R(s)$ is lossless negative imaginary if and only if

1) $\operatorname{det}(A) \neq 0, R(\infty)=R^{T}(\infty)$;

2) there exist matrices $X \in \mathbb{R}^{n \times n}, Y \in \mathbb{R}^{n \times m}$ such that $A^{T} X+$ $X^{T} A=0$ and (5)-(7) hold.

Proof: Similar to the proof of Theorem 1, Assumption 1 and $R(s) \in \mathcal{R}^{m \times m}$ imply that $N=0$. Hence, (8) holds. Then in view of [4, Theorem 1], one has that $R(s)$ is lossless negative imaginary if and only if the following conditions hold:

1) $\operatorname{det}\left(A_{1}\right) \neq 0, D-C_{2} B_{2}=\left(D-C_{2} B_{2}\right)^{T}$;

2) there exists a matrix $X_{1} \in \mathbb{R}^{n_{1} \times n_{1}}, X_{1}=X_{1}^{T}>0$ such that $A_{1}^{T} X_{1}+X_{1} A_{1}=0$ and $C_{1}^{T}+X_{1} A_{1}^{-1} B_{1}=0$.

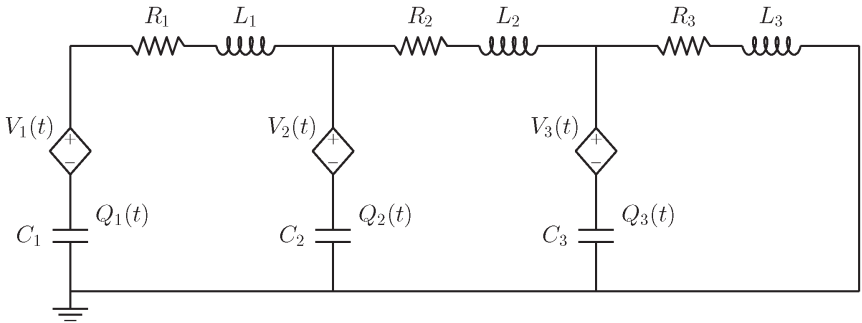

Fig. 1. Multiple-input multiple-output RLC circuit.

The above two conditions can be proved to be equivalent to the two conditions in this theorem by following similar lines as in the proof for Theorem 1 . The only differences between the two proofs are that the inequality signs " $\leq$ " in " $A^{T} X+X^{T} A \leq 0$ " and " $A_{1}^{T} X_{1}+X_{1} A_{1} \leq$ 0 " need to be replaced with equality signs " $=$ ".

Remark 6: Sufficiency in Theorem 1-3 also holds when weakening Assumption 1 to either $\left(N, C_{2}\right)$ being observable or $N B_{2}=0$, which allows the system to have impulse modes.

\section{ILlustrative EXAMPLE}

In this section, a multiple-input and multiple-output RLC network as shown in Fig. 1 is used to illustrate the negative imaginary lemma for descriptor systems.

Consider the circuit in Fig. 1. The inputs are the outputs of the controlled voltage sources $V_{i}(t)$, and the outputs are the charges on the capacitances $Q_{i}(t), i=1,2,3$. A system of ordinary differential equations can be constructed, and a standard state-space model can be used for the negative imaginary lemmas. However, a descriptor statespace system model is much more easily obtained by modified nodal analysis [13], [14]. The following matrices are constructed from the topology structure of the circuit network by modified nodal analysis:

$$
\begin{aligned}
A_{\mathrm{R}}=\left[\begin{array}{ccc}
1 & 0 & 0 \\
-1 & 0 & 0 \\
0 & 1 & 0 \\
0 & -1 & 0 \\
0 & 0 & 1 \\
0 & 0 & -1 \\
0 & 0 & 0 \\
0 & 0 & 0 \\
0 & 0 & 0
\end{array}\right], A_{\mathrm{L}}=\left[\begin{array}{ccc}
0 & 0 & 0 \\
1 & 0 & 0 \\
-1 & 0 & 0 \\
0 & 1 & 0 \\
0 & -1 & 0 \\
0 & 0 & 1 \\
0 & 0 & 0 \\
0 & 0 & 0 \\
0 & 0 & 0
\end{array}\right] \\
A_{\mathrm{C}}=\left[\begin{array}{ccc}
0 & 0 & 0 \\
0 & 0 & 0 \\
0 & 0 & 0 \\
0 & 0 & 0 \\
0 & 0 & 0 \\
0 & 0 & 0 \\
-1 & 0 & 0 \\
0 & -1 & 0 \\
0 & 0 & -1
\end{array}\right], A_{\mathrm{V}}=\left[\begin{array}{ccc}
-1 & 0 & 0 \\
0 & 0 & 0 \\
0 & -1 & 0 \\
0 & 0 & 0 \\
0 & 0 & -1 \\
0 & 0 & 0 \\
1 & 0 & 0 \\
0 & 1 & 0 \\
0 & 0 & 1
\end{array}\right] .
\end{aligned}
$$

After defining the resistance, inductance and capacitance matrices as follows, respectively,

$$
\begin{aligned}
& \mathrm{R}=\left[\begin{array}{ccc}
R_{1} & 0 & 0 \\
0 & R_{2} & 0 \\
0 & 0 & R_{3}
\end{array}\right], \quad \mathrm{L}=\left[\begin{array}{ccc}
L_{1} & 0 & 0 \\
0 & L_{2} & 0 \\
0 & 0 & L_{3}
\end{array}\right] \\
& \mathrm{C}=\left[\begin{array}{ccc}
C_{1} & 0 & 0 \\
0 & C_{2} & 0 \\
0 & 0 & C_{3}
\end{array}\right]
\end{aligned}
$$


a descriptor state-space system model (1) can be constructed, and the system matrices are given by

$$
\begin{aligned}
& E=\left[\begin{array}{ccc}
A_{\mathrm{C}} \mathrm{C} A_{\mathrm{C}}^{T} & 0 & 0 \\
0 & \mathrm{~L} & 0 \\
0 & 0 & 0
\end{array}\right], A=\left[\begin{array}{ccc}
-A_{\mathrm{R}} \mathrm{R}^{-1} A_{\mathrm{R}}^{T} & -A_{\mathrm{L}} & -A_{\mathrm{V}} \\
A_{\mathrm{L}}^{T} & 0 & 0 \\
A_{\mathrm{V}}^{T} & 0 & 0
\end{array}\right] \\
& B=\left[\begin{array}{lll}
0 & 0 & -I
\end{array}\right]^{T}, \quad C=\left[\begin{array}{lll}
-C A_{\mathrm{C}}^{T} & 0 & 0
\end{array}\right], \quad D=0 .
\end{aligned}
$$

In this model, the system state, the system input and the system output are, respectively,

$$
x(t)=\left[\begin{array}{c}
v(t) \\
i_{\mathrm{L}}(t) \\
i_{\mathrm{V}}(t)
\end{array}\right], \quad u(t)=\left[\begin{array}{c}
V_{1}(t) \\
V_{2}(t) \\
V_{3}(t)
\end{array}\right], \quad y(t)=\left[\begin{array}{l}
Q_{1}(t) \\
Q_{2}(t) \\
Q_{3}(t)
\end{array}\right] .
$$

Here $v(t) \in \mathbb{R}^{9}$ is a vector of node potentials, $i_{\mathrm{L}}(t) \in \mathbb{R}^{3}$ and $i_{\mathrm{V}}(t) \in$ $\mathbb{R}^{3}$ are vectors of currents through inductors and voltage sources, respectively. It can be verified that $\operatorname{rank}(A)=15, R(\infty)=0, E=$ $E^{T} \geq 0, A+A^{T} \leq 0$ and $E A^{-1} B=-C^{T}$. Hence a feasible solution to the inequalities in (4)-(7) is given by $X=I \in \mathbb{R}^{15 \times 15}$ and $Y=0 \in \mathbb{R}^{15 \times 3}$. Note that the solution is usually not unique. Using Theorem 1, it follows that the descriptor system is negative imaginary for any $R_{i}>0, L_{i}>0$ and $C_{i}>0, i=1,2,3$; that is, the corresponding transfer function satisfies the conditions in Definition 1.

\section{CONCLUSION}

In this technical note, negative imaginary properties have been studied for descriptor linear systems. Under the assumption of a minimal realization, necessary and sufficient conditions have been obtained in each of the negative imaginary lemma, the strict negative imaginary lemma and the lossless negative imaginary lemma. Finally, an example from RLC circuits was presented to illustrate the developed theory.

A limitation of this study is that the transfer functions have to be proper. The question of how to develop negative imaginary lemmas for descriptor systems with non-proper transfer functions demands a careful revision of the definitions of negative imaginary transfer functions, and will be considered in future research.

\section{REFERENCES}

[1] A. Lanzon and I. R. Petersen, "Stability robustness of a feedback interconnection of systems with negative imaginary frequency response," IEEE Trans. Autom. Control, vol. 53, no. 4, pp. 1042-1046, Apr. 2008.

[2] I. R. Petersen and A. Lanzon, "Feedback control of negative-imaginary systems," IEEE Control Syst. Mag., vol. 30, no. 5, pp. 54-72, 2010.
[3] J. Xiong, I. R. Petersen, and A. Lanzon, "A negative imaginary lemma and the stability of interconnections of linear negative imaginary systems," IEEE Trans. Autom. Control, vol. 55, no. 10, pp. 2342-2347, Oct. 2010.

[4] J. Xiong, I. R. Petersen, and A. Lanzon, "On lossless negative imaginary systems," Automatica, vol. 48, no. 6, pp. 1213-1217, 2012.

[5] J. Xiong, I. R. Petersen, and A. Lanzon, "Finite frequency negative imaginary systems," IEEE Trans. Autom. Control, vol. 57, no. 11, pp. $2917-$ 2922, Nov. 2012.

[6] Z. Song, A. Lanzon, S. Patra, and I. R. Petersen, "A negative-imaginary lemma without minimality assumptions and robust state-feedback synthesis for uncertain negative-imaginary systems," Syst. \& Control Lett., vol. 61, no. 12, pp. 1269-1276, 2012.

[7] A. Ferrante and L. Ntogramatzidis, "Some new results in the theory of negative imaginary systems with symmetric transfer matrix function," Automatica, vol. 49, no. 7, pp. 2138-2144, 2013.

[8] M. A. Mabrok, A. G. Kallapur, I. R. Petersen, and A. Lanzon, "Generalizing negative imaginary systems theory to include free body dynamics: Control of highly resonant structures with free body motion," IEEE Trans. Autom. Control, vol. 59, no. 10, pp. 2692-2707, 2014.

[9] C. Cai and G. Hagen, "Stability analysis for a string of coupled stable subsystems with negative imaginary frequency response," IEEE Trans. Autom. Control, vol. 55, no. 8, pp. 1958-1963, 2010.

[10] M. R. Opmeer, "Infinite-dimensional negative imaginary systems," IEEE Trans. Autom. Control, vol. 56, no. 12, pp. 2973-2976, 2011.

[11] T. Reis and T. Stykel, "PABTEC: Passivity-preserving balanced truncation for electrical circuits," IEEE Trans. Comput.-Aided Design Integr. Circuits Syst., vol. 29, no. 9, pp. 1354-1367, 2010.

[12] F. D. Freitas, N. Martins, S. L. Varricchio, J. Rommes, and F. C. Véliz, "Reduced-order transfer matrices from RLC network descriptor models of electric power grids," IEEE Trans. Power Syst., vol. 26, no. 4, pp. 19051916, 2011.

[13] J. Vlach and K. Singhal, Computer Methods for Circuit Analysis and Design. New York, NY, USA: Van Nostrand Reinhold, 1983.

[14] R. W. Freund, "The SPRIM algorithm for structure-preserving order reduction of general RCL circuits," in Model Reduction for Circuit Simulation. New York, NY, USA: Springer, 2011, ch. 2, pp. 25-52.

[15] M. A. Mabrok, A. G. Kallapur, I. R. Petersen, and A. Lanzon, "Generalized negative imaginary lemma for descriptor systems," J. Mechan. Eng. and Automat., vol. 2, no. 1, pp. 17-21, 2012.

[16] P. Benner and M. Voigt, "Spectral characterization and enforcement of negative imaginariness for descriptor systems," Linear Alg. and its Applicat., vol. 439, no. 4, pp. 1104-1129, 2013.

[17] I. Masubuchi, "Dissipativity inequalities for continuous-time descriptor systems with applications to synthesis of control gains," Syst. \& Control Lett., vol. 55, no. 2, pp. 158-164, 2006.

[18] L. Dai, Singular Control Systems. New York, NY, USA: SpringerVerlag, 1989.

[19] R. W. Freund and F. Jarre, "An extension of the positive real lemma to descriptor systems," Optimiz. Methods and Softw., vol. 19, no. 1, pp. 69-87, 2004. 